\title{
DEPTH OF FACTORS OF SQUARE FREE MONOMIAL IDEALS
}

\author{
DORIN POPESCU
}

(Communicated by Irena Peeva)

\begin{abstract}
Let $I$ be an ideal of a polynomial algebra over a field generated by $r$ square free monomials of degree $d$. If $r$ is bigger than (or equal to, if $I$ is not principal) the number of square free monomials of $I$ of degree $d+1$, then $\operatorname{depth}_{S} I=d$. Let $J \subsetneq I, J \neq 0$ be generated by square free monomials of degree $\geq d+1$. If $r$ is bigger than the number of square free monomials of $I \backslash J$ of degree $d+1$ or, more generally, the Stanley depth of $I / J$ is $d$, then $\operatorname{depth}_{S} I / J=d$. In particular, Stanley's Conjecture holds in these cases.
\end{abstract}

\section{INTRODUCTION}

Let $S=K\left[x_{1}, \ldots, x_{n}\right]$ be the polynomial algebra in $n$ variables over a field $K$, $d$ a positive integer and $I \supsetneq J$ two square free monomial ideals of $S$ such that $I$ is generated in degrees $\geq d$, respectively $J$ in degrees $\geq d+1$. Let $\rho_{d}(I)$ be the number of all square free monomials of degree $d$ of $I$. It is easy to note (see Lemma 1.1) that $\operatorname{depth}_{S} I / J \geq d$. Our Theorem 2.2 gives a sufficient condition which implies $\operatorname{depth}_{S} I / J=d$; namely this happens when

$$
\rho_{d}(I)>\rho_{d+1}(I)-\rho_{d+1}(J) .
$$

Suppose that this condition holds. Then the Stanley depth of $I / J$ (see [1], 2], or here Remark 2.6) is $d$, and if Stanley's Conjecture holds, then $\operatorname{depth}_{S} I / J \leq d$, that is, the missing inequality. Thus to test Stanley's Conjecture means to test the equality $\operatorname{depth}_{S} I / J=d$, which is much easier since there exist very good algorithms to compute $\operatorname{depth}_{S} I / J$ but not so good to compute the Stanley depth of $I / J$. After many examples computed with the computer algebra system SINGULAR we understood that a result such as Theorem 2.2 is believable. The above condition is not necessary as Example 2.4 shows. Necessary and sufficient conditions could possibly be found for classifying some posets (see Remark 2.5), but this is not the subject of this paper.

The proof of Theorem 2.2 uses the Koszul homology and can be read without other preparation. Our first section gives easy proofs in special cases, but they are mainly an introduction to the subject. Remark 1.7 shows that Koszul homology seems to be the best tool for our problems. Section 2 starts with one example, where we give the idea of the proof of Theorem 2.2

Received by the editors June 3, 2012 and, in revised form, July 12, 2012.

2010 Mathematics Subject Classification. Primary 13C15; Secondary 13F20, 13F55, 13 P10.

Key words and phrases. Monomial ideals, depth, Stanley depth.

The author's support from grant ID-PCE-2011-1023 of the Romanian Ministry of Education, Research and Innovation is gratefully acknowledged. 
If $I$ is generated by more (or equal, if $I$ is not principal) square free monomials of degree $d$ than $\left(\begin{array}{c}n \\ d+1\end{array}\right)$, or more general than $\rho_{d+1}(I)$, then $\operatorname{depth}_{S} I=d$ as is shown in our Corollary 3.4. This extends [9, Corollary 3], which was the starting point of our research, the proof there being easier. Remark 3.5 says that the condition of Corollary 3.4 is tight.

The conditions above are reasons for the fact that the Stanley depth (sdepth) ${ }_{S} I / J$ is $d$, as we explain in Remark 2.6, and we see that they imply $\operatorname{depth}_{S} I / J=d$. But what happens if we just suppose that $\operatorname{sdepth}_{S} I / J=d$ ? Then there exists a monomial square free ideal $I^{\prime} \subset I$ such that $\rho_{d}\left(I^{\prime}\right)>\rho_{d+1}\left(I^{\prime}\right)-\rho_{d+1}\left(I^{\prime} \cap J\right)$ using our Theorem 4.1 (somehow an extension of [10, Lemma 3.3]), and it follows also that $\operatorname{depth}_{S} I / J=d$ by our Theorem 4.3 .

\section{FACTORS OF SQUARE FREE MONOMIAL IDEALS}

Let $J \subsetneq I$ be two nonzero square free monomial ideals of $S$ and $d$ a positive integer. Let $\rho_{d}(I)$ be the number of all square free monomials of degree $d$ of I. Suppose that $I$ is generated by square free monomials $f_{1}, \ldots, f_{r}, r>0$, of degrees $\geq d$ and $J$ is generated by square free monomials of degree $\geq d+1$. Set $s:=\rho_{d+1}(I)-\rho_{d+1}(J)$ and let $b_{1}, \ldots, b_{s}$ be the square free monomials of $I \backslash J$ of degree $d+1$.

Lemma 1.1. $\operatorname{depth}_{S} I, \operatorname{depth}_{S} I / J \geq d$.

Proof. By [2, Proposition 3.1] we have $\operatorname{depth}_{S} I \geq d$, $\operatorname{depth}_{S} J \geq d+1$. The conclusion follows from applying the Depth Lemma in the exact sequence $0 \rightarrow J \rightarrow$ $I \rightarrow I / J \rightarrow 0$.

Lemma 1.2. Suppose that $J=E+F, F \not \subset E$, where $E, F$ are ideals generated by square free monomials of degree $d+1$, respectively $>d+1$. Then $\operatorname{depth}_{S} I / J=d$ if and only if $\operatorname{depth}_{S} I / E=d$.

Proof. We may suppose that in $E$ there exists no monomial generator of $F$. In the exact sequence

$$
0 \rightarrow J / E \rightarrow I / E \rightarrow I / J \rightarrow 0
$$

we see that the first end is isomorphic with $F /(F \cap E)$ and has depth $\geq d+2$ by Lemma 1.1. Apply the Depth Lemma and we are done.

Before trying to extend the above lemma it is useful to see the next example.

Example 1.3. Let $n=4, d=1, I=\left(x_{2}\right), E=\left(x_{2} x_{4}\right), F=\left(x_{1} x_{2} x_{3}\right)$. Then $\operatorname{depth}_{S} I / E=3$ and $\operatorname{depth}_{S} I /(E+F)=2$.

Lemma 1.4. Let $H$ be an ideal generated by square free monomials of degrees $d+1$. Then $\operatorname{depth}_{S} I / J=d$ if and only if $\operatorname{depth}_{S}(I+H) / J=d$.

Proof. By induction on the number of the generators of $H$ it is enough to consider the case $H=(u)$ for some square free monomial $u \notin I$ of degrees $d+1$. In the exact sequence

$$
0 \rightarrow I / J \rightarrow(I+(u)) / J \rightarrow(I+(u)) / I \rightarrow 0
$$

we see that the last term is isomorphic with $(u) / I \cap(u)$ and has depth $\geq d+1$ by Lemma 1.1 since $I \cap(u)$ has only monomials of degrees $>d+1$. Using the Depth Lemma the first term has depth $d$ if and only if the middle has depth $d$, which is enough. 
Using Lemmas 1.2 1.4 we may suppose always in our consideration that $I, J$ are generated in degree $d$, respectively $d+1$; in particular, $f_{i}$ have degree $d$.

Lemma 1.5. Let $e \leq r$ be a positive integer and $I^{\prime}=\left(f_{1}, \ldots, f_{e}\right), J^{\prime}=J \cap I^{\prime}$. If $\operatorname{depth}_{S} I^{\prime} / J^{\prime}=d$, then $\operatorname{depth}_{S} I / J=d$.

Proof. In the exact sequence

$$
0 \rightarrow I^{\prime} / J^{\prime} \rightarrow I / J \rightarrow I /\left(I^{\prime}+J\right) \rightarrow 0
$$

the right end has depth $\geq d$ by Lemma 1.1 because

$$
I /\left(I^{\prime}+J\right) \cong\left(f_{e+1}, \ldots, f_{r}\right) /\left(\left(J+I^{\prime}\right) \cap\left(f_{e+1}, \ldots, f_{r}\right)\right)
$$

and $\left(J+I^{\prime}\right) \cap\left(f_{e+1}, \ldots, f_{r}\right)$ is generated by monomials of degree $>d$. If the left end has depth $d$, then the middle has the same depth by the Depth Lemma.

Lemma 1.6. Suppose that there exists $i \in[r]$ such that $f_{i}$ has in $J$ all square free multiples of degree $d+1$. Then $\operatorname{depth}_{S} I / J=d$.

Proof. We may suppose $i=1$. By our hypothesis $J: f_{1}$ is generated by $(n-d)$ variables. If $r=1$, then the depth of $I / J \cong S /\left(J: f_{1}\right)$ is $d$. If $r>1$, apply the above lemma for $e=1$.

Remark 1.7. Suppose in the proof of the above lemma that $f_{1}=x_{1} \cdots x_{d}$. Then the hypothesis says that $\left(x_{d+1}, \ldots, x_{n}\right) f_{1} \subset J$. It follows that $z=f_{1} e_{\sigma_{1}}, e_{\sigma_{1}}=e_{d+1} \wedge$ $\ldots \wedge e_{n}$ induces a nonzero element in the Koszul homology module $H_{n-d}(x ; I / J)$ of $I / J$ (some details from Koszul homology theory are given in Example 2.1). Thus $\operatorname{depth}_{S} I / J \leq d$ by [1, Theorem 1.6.17]; the other inequality follows from Lemma 1.1. This gives a different proof of the above lemma using stronger tools, which will be very useful in the next section. We also recall that $H_{n-d}(x ; I / J) \cong$ $\operatorname{Tor}_{n-d}^{S}(K, I / J) \neq(0)$ gives $\operatorname{pd}_{S} I / J \geq n-d$, which means $\operatorname{depth}_{S} I / J \leq d$ by the Auslander-Buchsbaum Theorem [1, Theorem 1.3.3].

Lemma 1.8. Suppose that $d=1, f_{i}=x_{i}, i \in[r]$, and $b_{j} \in S^{\prime}=K\left[x_{1}, \ldots, x_{r}\right]$ for all $j \in[s]$. Then $\operatorname{depth}_{S} I / J=1$ independently of $r, s$ ( $s$ may be greater than $r$ ).

Proof. Set $I^{\prime}=I \cap S^{\prime}$ and $J^{\prime}=J \cap S^{\prime}$. Then $\operatorname{depth}_{S^{\prime}} S^{\prime} / I^{\prime}=0$ and $\operatorname{depth}_{S^{\prime}} S^{\prime} / J^{\prime}>0$ by Lemma 1.1. From the exact sequence

$$
0 \rightarrow I^{\prime} / J^{\prime} \rightarrow S^{\prime} / J^{\prime} \rightarrow S^{\prime} / I^{\prime} \rightarrow 0
$$

it follows that $\operatorname{depth}_{S^{\prime}} I^{\prime} / J^{\prime}=1$ by the Depth Lemma. If $r<n$, then note that $\left(x_{r+1}, \ldots, x_{n}\right) I \subset J$, and so $\operatorname{depth}_{S} I / J=\operatorname{depth}_{S}\left(I^{\prime} S / J^{\prime} S\right)-(n-r)=$ $\operatorname{depth}_{S^{\prime}} I^{\prime} / J^{\prime}=1$.

Proposition 1.9. Suppose that $d=1$ and $r>s$. Then $\operatorname{depth}_{S} I / J=1$.

Proof. By Lemma 1.8 we may suppose that $I=\left(x_{1}, \ldots, x_{r}\right)$ with $r<n$. Using Lemma 1.6 we may suppose that each $x_{i}, i \in[r]$, divides a certain $b_{k}$. Apply induction on $s$; the case $s=0$ follows from Lemma 1.6. Assume that $s>0$. We may suppose that each $b_{k}$ is a product of two different $x_{i}, i \in[r]$, because if let us say $b_{s}$ is just a multiple of one $x_{i}, i \in[r]$, for example $x_{r}$, then we may take $I^{\prime}=\left(x_{1}, \ldots, x_{r-1}\right), J^{\prime}=J \cap I^{\prime}$ and we get $\operatorname{depth}_{S} I^{\prime} / J^{\prime}=1$ by induction hypothesis on $s$ since $r-1>s-1$; that is, $\operatorname{depth}_{S} I / J=1$ by Lemma 1.5. But if each $b_{k}$ is a product of two different $x_{i}, i \in[r]$, we see that $b_{j} \in S^{\prime}=K\left[x_{1}, \ldots, x_{r}\right]$ for all $j \in[s]$ and we may again apply Lemma 1.8 


\section{MAIN RESUlT}

We want to extend Proposition 1.9 for the case $d>1$. The next example is an illustration of our method.

Example 2.1. Let $n=6, d=2, f_{1}=x_{1} x_{6}, f_{2}=x_{1} x_{5}, f_{3}=x_{1} x_{3}, f_{4}=x_{3} x_{4}$, $f_{5}=x_{2} x_{4}$,

$$
\begin{gathered}
J=\left(x_{1} x_{2} x_{4}, x_{1} x_{2} x_{5}, x_{1} x_{2} x_{3}, x_{1} x_{2} x_{6}, x_{1} x_{3} x_{6}, x_{1} x_{4} x_{5}, x_{1} x_{4} x_{6},\right. \\
\left.x_{2} x_{4} x_{5}, x_{2} x_{4} x_{6}, x_{3} x_{4} x_{5}, x_{3} x_{4} x_{6}\right)
\end{gathered}
$$

and $I=\left(f_{1}, f_{2}, f_{3}, f_{4}, f_{5}\right)$. We have $s=4, b_{1}=x_{5} f_{1}=x_{6} f_{2}, b_{2}=x_{3} f_{2}=x_{5} f_{3}$, $b_{3}=x_{4} f_{3}=x_{1} f_{4}, b_{4}=x_{2} f_{4}=x_{3} f_{5}$. Let $\partial_{i}: K_{i}(x ; I / J) \rightarrow K_{i-1}(x ; I / J)$, $K_{i}(x ; I / J) \cong S^{\left(\begin{array}{c}6 \\ i\end{array}\right)}, i \in[6]$, be the Koszul derivation given by

$$
\partial_{i}\left(e_{j_{1}} \wedge \ldots \wedge e_{j_{i}}\right)=\sum_{k=1}^{i}(-1)^{k+1} x_{j_{k}} e_{j_{1}} \wedge \ldots \wedge e_{j_{k-1}} \wedge e_{j_{k+1}} \wedge \ldots \wedge e_{j_{i}}
$$

We consider the following elements of $K_{4}(x ; I / J)$ :

$$
\begin{gathered}
e_{\sigma_{1}}=e_{2} \wedge \ldots \wedge e_{5}, e_{\sigma_{2}}=e_{2} \wedge \ldots \wedge e_{4} \wedge e_{6}, e_{\sigma_{3}}=e_{2} \wedge e_{4} \wedge \ldots \wedge e_{6}, \\
e_{\sigma_{4}}=e_{1} \wedge e_{2} \wedge e_{5} \wedge e_{6}, e_{\sigma_{5}}=e_{1} \wedge e_{3} \wedge e_{5} \wedge e_{6} .
\end{gathered}
$$

Then the element

$$
z=f_{1} e_{\sigma_{1}}-f_{2} e_{\sigma_{2}}-f_{3} e_{\sigma_{3}}-f_{4} e_{\sigma_{4}}+f_{5} e_{\sigma_{5}}
$$

satisfies

$$
\begin{gathered}
\partial_{4}(z)=\left(-b_{1}+b_{1}\right) e_{2} \wedge e_{3} \wedge e_{4}+\left(b_{2}-b_{2}\right) e_{2} \wedge e_{4} \wedge e_{6} \\
+\left(b_{3}-b_{3}\right) e_{2} \wedge e_{5} \wedge e_{6}+\left(b_{4}-b_{4}\right) e_{1} \wedge e_{5} \wedge e_{6}=0,
\end{gathered}
$$

since $\left(b_{k}\right)$ are the only monomials of degree 3 which are not in $J$. Note that in a term $u e_{\sigma}$ of an element from $\operatorname{Im} \partial_{5}$ we have $u$ of degree $\geq 3$ because $I$ is generated in degree 2. Thus $z \notin \operatorname{Im} \partial_{5}$ induces a nonzero element in $H_{4}(x ; I / J)$. By [1, Theorem 1.6.17] we get $\operatorname{depth}_{S} I / J \leq 2$, which is enough.

Theorem 2.2. If $r>s$, then $\operatorname{depth}_{S} I / J=d$, independently of the characteristic of $K$.

Proof. Let $\operatorname{supp} f_{i}=\left\{j \in[n]: x_{j} \mid f_{i}\right\}, e_{\sigma_{i}}=\bigwedge_{j \in\left([n] \backslash \operatorname{supp} f_{i}\right)} \quad e_{j}$ and $e_{\tau_{k}}=$ $\bigwedge_{j \in\left([n] \backslash \operatorname{supp} b_{k}\right)} e_{j}$. By [1, Theorem 1.6.17] it is enough to show, as in the above example, that there exist $y_{i} \in K, i \in[r]$, such that $z=\sum_{i=1}^{r} y_{i} f_{i} e_{\sigma_{i}}$ induces a nonzero element of $H_{n-d}(x ; I / J)$. Let $\partial_{i}$ be the Koszul derivation as above. Then

$$
\partial_{n-d}(z)=\sum_{k=1}^{s}\left(\sum_{i \in[r], f_{i} \mid b_{k}} \varepsilon_{k i} y_{i}\right) b_{k} e_{\tau_{k}}
$$

for some $\varepsilon_{k i} \in\{1,-1\}$. Thus $\partial_{n-d}(z)=0$ if and only if $\sum_{i \in[r], f_{i} \mid b_{k}} \varepsilon_{k i} y_{i}=0$ for all $k \in[s]$. This is a system of $s$ homogeneous linear equations in $r$ variables $Y$ which must have a nonzero solution in $K$ because $r>s$. As in the above example, $z \notin \operatorname{Im} \partial_{n-d+1}$ if $I$ is generated in degree $d$ (this may be supposed by Lemmas 1.2. 1.4).

The condition given in Theorem 2.2 is tight as is shown in the following two examples. 
Example 2.3. Let $n=4, d=2, f_{1}=x_{1} x_{3}, f_{2}=x_{2} x_{4}, f_{3}=x_{1} x_{4}$ and $I=$ $\left(f_{1}, \ldots, f_{3}\right), J=\left(x_{2} x_{3} x_{4}\right)$ be ideals of $S$. We have $r=s=3, b_{1}=x_{1} x_{2} x_{3}$, $b_{2}=x_{1} x_{2} x_{4}, b_{3}=x_{1} x_{3} x_{4}$, and $\operatorname{depth}_{S} I / J=d+1$.

Example 2.4. Let $n=6, d=2, f_{1}=x_{1} x_{5}, f_{2}=x_{2} x_{3}, f_{3}=x_{3} x_{4}, f_{4}=x_{1} x_{6}$, $f_{5}=x_{1} x_{4}, f_{6}=x_{1} x_{2}$, and $I=\left(f_{1}, \ldots, f_{6}\right)$,

$$
J=\left(x_{1} x_{2} x_{4}, x_{1} x_{2} x_{5}, x_{1} x_{3} x_{5}, x_{1} x_{3} x_{6}, x_{1} x_{4} x_{6}, x_{2} x_{3} x_{5}, x_{2} x_{3} x_{6}, x_{3} x_{4} x_{5}, x_{3} x_{4} x_{6}\right) .
$$

We have $r=s=6$ and $b_{1}=x_{1} x_{4} x_{5}, b_{2}=x_{2} x_{3} x_{4}, b_{3}=x_{1} x_{2} x_{3}, b_{4}=x_{1} x_{5} x_{6}$, $b_{5}=x_{1} x_{3} x_{4}, b_{6}=x_{1} x_{2} x_{6}$, but $\operatorname{depth}_{S} I / J=2($ although $d=2)$.

Remark 2.5. Example 2.4 shows that one could find a nice class of factors of square free monomial ideals with $r=s$ but $\operatorname{depth}_{S} I / J=d$, similarly as in 9, Lemma 6]. An important tool seems to be a classification of the possible posets given on $f_{1}, \ldots, f_{r}, b_{1}, \ldots, b_{s}$ by the divisibility.

Remark 2.6. Given $J \subsetneq I$ two square free monomial ideals of $S$ as above, one can consider the poset $P_{I \backslash J}$ of all square free monomials of $I \backslash J$ (a finite set) with the order given by the divisibility. Let $\mathcal{P}$ be a partition of $P_{I \backslash J}$ in intervals $[u, v]=\left\{w \in P_{I \backslash J}: u|w, w| v\right\}$, let us say $P_{I \backslash J}=\bigcup_{i}\left[u_{i}, v_{i}\right]$, the union being disjoint. Define $\operatorname{sdepth} \mathcal{P}=\min _{i} \operatorname{deg} v_{i}$ and $\operatorname{sdepth}_{S} I / J=\max _{\mathcal{P}} \operatorname{sdepth} \mathcal{P}$, where $\mathcal{P}$ runs in the set of all partitions of $P_{I \backslash J}$. This is the Stanley depth of $I / J$; in fact this is an equivalent definition (see [11, [2]). If $r>s$, then it is obvious that $\operatorname{sdepth}_{S} I / J=d$, and so Theorem 2.2 says that Stanley's Conjecture holds, that is, $\operatorname{sdepth}_{S} I / J \geq \operatorname{depth}_{S} I / J$. "In general the Stanley depth of a monomial ideal $I$ is greater than or equal with the Lyubeznik" size of $I$ increased by one (see [3]). Stanley's Conjecture holds for intersections of four monomial prime ideals of $S$ by [ 5 ] and [7] and for square free monomial ideals of $K\left[x_{1}, \ldots, x_{5}\right]$ by [6] (a short exposition on this subject is given in [8]). Also, Stanley's Conjecture holds for intersections of three monomial primary ideals by [13. In the case of a nonsquare free monomial ideal $I$ a useful inequality is $\operatorname{sdepth} I \leq \operatorname{sdepth} \sqrt{I}$ (see [4, Theorem 2.1]).

\section{Around Theorem 2.2}

Let $S^{\prime}=K\left[x_{1}, \ldots, x_{n-1}\right]$ be a polynomial ring in $n-1$ variables over a field $K$, let $S=S^{\prime}\left[x_{n}\right]$ and let $U, V \subset S^{\prime}, V \subset U$ be two square free monomial ideals. Set $W=\left(V+x_{n} U\right) S$. Actually, every monomial square free ideal $T$ of $S$ has this form because then $\left(T: x_{n}\right)$ is generated by an ideal $U \subset S^{\prime}$ and $T=\left(V+x_{n} U\right) S$ for $V=T \cap S^{\prime}$.

Lemma 3.1 ([6]). Suppose that $U \neq V$ and $\operatorname{depth}_{S^{\prime}} S^{\prime} / U=\operatorname{depth}_{S^{\prime}} S^{\prime} / V=$ $\operatorname{depth}_{S^{\prime}} U / V$. Then $\operatorname{depth}_{S} S / W=\operatorname{depth}_{S^{\prime}} S^{\prime} / U$.

Lemma 3.2. Suppose that $U \neq V$ and $d:=\operatorname{depth}_{S^{\prime}} S^{\prime} / U=\operatorname{depth}_{S^{\prime}} S^{\prime} / V$. Then $d=\operatorname{depth}_{S^{\prime}} U / V$ if and only if $d=\operatorname{depth}_{S} S / W$.

Proof. The necessity follows from the above lemma. For sufficiency note that in the exact sequence

$$
0 \rightarrow V S \rightarrow W \rightarrow U S / V S \rightarrow 0
$$

the depth of the left end is $d+2$ and the middle term has depth $d+1$. It follows that $\operatorname{depth}_{S} U S / V S=d+1$ by the Depth Lemma, which is enough. 
Let $I$ be an ideal of $S$ generated by square free monomials of degree $\geq d$ and $x_{n} f_{1}, \ldots, x_{n} f_{r}, r>0$, be the square free monomials of $I \cap\left(x_{n}\right)$ of degree $d$. Set $U=\left(f_{1}, \ldots, f_{r}\right), V=I \cap S^{\prime}$.

Theorem 3.3. If $r>\rho_{d}(U)-\rho_{d}(U \cap V)$, then $\operatorname{depth}_{S} S / I=\operatorname{depth}_{S^{\prime}}(U+V) / V=$ $d-1$.

Proof. By Theorem 2.2 we have $\operatorname{depth}_{S^{\prime}}(U+V) / V=\operatorname{depth}_{S^{\prime}} U /(U \cap V)=d-1$. Using Lemmas 1.2, 1.4 we get

$$
\operatorname{depth}_{S^{\prime}}(U+V) / V=\operatorname{depth}_{S^{\prime}}\left(\left(I: x_{n}\right) \cap S^{\prime}\right) /\left(I \cap S^{\prime}\right)=d-1 .
$$

If $\operatorname{depth}_{S^{\prime}} S^{\prime} /\left(I \cap S^{\prime}\right)=\operatorname{depth}_{S^{\prime}} S^{\prime} /\left(\left(I: x_{n}\right) \cap S^{\prime}\right)=d-1$, then $\operatorname{depth}_{S} S / I=d-1$ by Lemma 3.2. If $\operatorname{depth}_{S^{\prime}} S^{\prime} /\left(\left(I: x_{n}\right) \cap S^{\prime}\right)=d-2$, then in the exact sequence

$$
0 \rightarrow S /\left(I: x_{n}\right) \stackrel{x_{n}}{\longrightarrow} S / I \rightarrow S^{\prime} /\left(I \cap S^{\prime}\right) \rightarrow 0
$$

the first term has depth $d-1$ and the other two have depth $\geq d-1$ by Lemma 1.1 . By the Depth Lemma it follows that $\operatorname{depth}_{S} S / I=d-1$.

It remains to consider the case when at least one of $\operatorname{depth}_{S^{\prime}} S^{\prime} /\left(\left(I: x_{n}\right) \cap S^{\prime}\right)$ and $\operatorname{depth}_{S^{\prime}} S^{\prime} /\left(I \cap S^{\prime}\right)$ is $\geq d$. Using the Depth Lemma in the exact sequence

$$
0 \rightarrow\left(\left(I: x_{n}\right) \cap S^{\prime}\right) /\left(I \cap S^{\prime}\right) \rightarrow S^{\prime} /\left(I \cap S^{\prime}\right) \rightarrow S^{\prime} /\left(\left(I: x_{n}\right) \cap S^{\prime}\right) \rightarrow 0
$$

we see that necessarily the depth of the last term is $\geq d$ and the depth of the middle term is $d-1$. But then the Depth Lemma applied to the previous exact sequence gives $\operatorname{depth}_{S} S / I=d-1$ too.

The following corollary extends [9, Corollary 3].

Corollary 3.4. Let $I$ be an ideal generated by $\mu(I)>1$ square free monomials of degree $d$. If $\mu(I) \geq \rho_{d+1}(I)$, in particular if $\mu(I) \geq\left(\begin{array}{c}n \\ d+1\end{array}\right)$, then $\operatorname{depth}_{S} I=d$.

Proof. We have $I=\left(V+x_{n}(U+V)\right) S$ as above. Renumbering the variables we may suppose that $U, V \neq 0$. Note that $\mu(I)=r+\rho_{d}(V)$ and $\rho_{d+1}(I)=$ $\rho_{d+1}(V)+\rho_{d}(U+V)>\rho_{d}(V)+\rho_{d}(U)-\rho_{d}(U \cap V)$. By hypothesis, $\mu(I) \geq \rho_{d+1}(I)$, and so $r>\rho_{d}(U)-\rho_{d}(U \cap V)$. Applying Theorem 3.3 we get $\operatorname{depth}_{S} S / I=d-1$, which is enough.

Remark 3.5. In Example 2.3 take $S^{\prime}=K\left[x_{1}, \ldots, x_{5}\right]$ and $L=\left(J+x_{5} I\right) S^{\prime}$. We have $\mu(L)=4<\left(\begin{array}{c}5 \\ 3+1\end{array}\right)$; that is, the hypothesis of the above corollary is not fulfilled. This is the reason that $\operatorname{depth}_{S^{\prime}} L=4$ by Lemma 3.2 since $\operatorname{depth}_{S} I / J=3$. Thus the condition of the above corollary is tight.

\section{Minimal Stanley Depth}

Let $S=K\left[x_{1}, \ldots, x_{n}\right]$ be the polynomial algebra in $n$-variables over a field $K$, $d$ a positive integer and $J \subsetneq I$ two square free monomial ideals of $S$. Let $\rho_{d}(I)$ be the number of all square free monomials of degree $d$ of $I$. Suppose that $\rho_{d}(I)>0$ and $I$ is generated in degree $\geq d$. It follows that $\operatorname{sdepth}_{S} I / J \geq d$.

Theorem 4.1. The following statements are equivalent:

(1) $\operatorname{sdepth}_{S} I / J=d$,

(2) there exist some square free monomials of degree $d$ in I which generate an ideal $I^{\prime}$ such that $\rho_{d}\left(I^{\prime}\right)>\rho_{d+1}\left(I^{\prime}\right)-\rho_{d+1}\left(I^{\prime} \cap J\right)$. 
Proof. If $J \neq 0$, then $J$ is generated in degree $\geq d$; we may even suppose that $J$ is generated in degree $\geq d+1$ using an easy isomorphism. Let $\mathcal{M}_{d}(I)$ be the set of all square free monomials of $I$ of degree $d$ and $\mathcal{B}=\mathcal{M}_{d+1} \backslash J$. We consider the bipartite graph $G$ defined by $V(G)=\mathcal{M}_{d}(I) \cup \mathcal{B}$; an edge of $G$ can have only endpoints $f \in \mathcal{M}_{d}(I)$ and $b \in \mathcal{B}$ with $f \mid b$. Given $f \in \mathcal{M}_{d}(I)$ let $\Gamma(f)$ be the set of all vertices $b$ adjacent to $f$, and for $A \subset \mathcal{M}_{d}(I)$ set $\Gamma(A)=\bigcup_{f \in A} \Gamma(f)$. By P. Hall's marriage theorem [12 there is a complete matching from $\mathcal{M}_{d}(I)$ to $\mathcal{B}$ if and only if $|\Gamma(A)| \geq|A|$ for every subset $A \subset \mathcal{M}_{d}(I)$. Thus $\operatorname{sdepth}_{S} I / J=d$ if and only if there exists no complete matching above, and so there exists a subset $A \subset \mathcal{M}_{d}(I)$ such that $|\Gamma(A)|<|A|$; that is, $I^{\prime}=(A)$ satisfies the second statement.

For $J=0$ we get the following corollary, which is close to [10, Lemma 3.3].

Corollary 4.2. The following statements are equivalent:

(1) $\operatorname{sdepth}_{S} I=d$,

(2) there exist some square free monomials of degree $d$ in I which generate an ideal $I^{\prime}$ such that $\rho_{d}\left(I^{\prime}\right)>\rho_{d+1}\left(I^{\prime}\right)$.

Theorem 4.3. If $\operatorname{sdepth}_{S} I / J=d$, then $\operatorname{depth}_{S} I / J=d$; that is, Stanley's conjecture holds in this case.

Proof. By Theorem 4.1 there exists a monomial square free ideal $I^{\prime} \subset I$ such that $\rho_{d}\left(I^{\prime}\right)>\rho_{d+1}\left(I^{\prime}\right)-\rho_{d+1}\left(I^{\prime} \cap J\right)$. Then $\operatorname{depth}_{S} I^{\prime} /\left(I^{\prime} \cap J\right)=d$ by Theorem 2.2 (if $J=0$ we apply Corollary 3.4). Now it is enough to apply Lemma 1.5.

\section{ACKNOWLEDGEMENT}

The author thanks a referee who found gaps in a preliminary version of this paper.

\section{REFERENCES}

[1] Winfried Bruns and Jürgen Herzog, Cohen-Macaulay rings, Cambridge Studies in Advanced Mathematics, vol. 39, Cambridge University Press, Cambridge, 1993. MR1251956 (95h:13020)

[2] Jürgen Herzog, Marius Vladoiu, and Xinxian Zheng, How to compute the Stanley depth of a monomial ideal, J. Algebra 322 (2009), no. 9, 3151-3169, DOI 10.1016/j.jalgebra.2008.01.006. MR2567414 (2010k:13036)

[3] Jürgen Herzog, Dorin Popescu, and Marius Vladoiu, Stanley depth and size of a monomial ideal, Proc. Amer. Math. Soc. 140 (2012), no. 2, 493-504, DOI 10.1090/S0002-9939-201111160-2. MR2846317 (2012h:13024)

[4] Muhammad Ishaq, Upper bounds for the Stanley depth, Comm. Algebra 40 (2012), no. 1, 87-97, DOI 10.1080/00927872.2010.523642. MR.2876290

[5] Adrian Popescu, Special Stanley decompositions, Bull. Math. Soc. Sci. Math. Roumanie (N.S.) 53(101) (2010), no. 4, 363-372. MR2777680(2012a:13037)

[6] Dorin Popescu, An inequality between depth and Stanley depth, Bull. Math. Soc. Sci. Math. Roumanie (N.S.) 52(100) (2009), no. 3, 377-382. MR2554495 (2010h:13019)

[7] D. Popescu, Stanley conjecture on intersections of four monomial prime ideals, Communications in Algebra 41 (2013), no. 11, 4351-4362. MR3169523

[8] Dorin Popescu, Bounds of Stanley depth, An. Ştiinţ. Univ. "Ovidius" Constanţa Ser. Mat. 19 (2011), no. 2, 187-194. MR2794292 (2012d:13038)

[9] Dorin Popescu, Depth and minimal number of generators of square free monomial ideals, An. Ştiinţ. Univ. "Ovidius" Constanţa Ser. Mat. 19 (2011), no. 3, 163-166. MR2879095

[10] Y.H. Shen, When will the Stanley depth increase? Proc. Amer. Math. Soc. 141 (2013), no. 7, 2265-2274. MR 3043008 
[11] Richard P. Stanley, Linear Diophantine equations and local cohomology, Invent. Math. 68 (1982), no. 2, 175-193, DOI 10.1007/BF01394054. MR666158(83m:10017)

[12] J. H. van Lint and R. M. Wilson, A course in combinatorics, 2nd ed., Cambridge University Press, Cambridge, 2001. MR.1871828 (2002i:05001)

[13] A. Zarojanu, Stanley conjecture on intersections of three monomial primary ideals, Bull. Math. Soc. Sc. Math. Roumanie (N.S.) 55(103) (2012), no. 3, 335-339. MR2987069

Simion Stoilow Institute of Mathematics of Romanian Academy, Research Unit 5 , P.O. Box 1-764, Bucharest 014700, Romania

E-mail address: dorin.popescu@imar.ro 